nature

immunology

\title{
New dimensions in immunology
}

\section{Emerging technologies are broadening our understanding of the human immune system, but capitalizing on their application will likely require philosophical and practical changes to the way research is done.}

B asic research studies in inbred animals have proven indispensable for unraveling the molecular underpinnings of the immune system. We have made less progress, however, in understanding the workings of the human immune system. All too often, animal models fail to faithfully recapitulate all the facets of a human disease, and therapies that work well in preclinical models fail to show efficacy in human trials. Now, a host of high-throughput technologies are enabling characterization of human immune cells in unprecedented detail and depth. But to truly galvanize human research studies, we must not only overcome numerous technological hurdles but also change the way immunological research itself is organized and implemented.

One of the most formidable obstacles facing research into the human immune system is its much greater interindividual and intraindividual diversity compared with that of inbred mice. One can take a group of animals in a pathogen-free facility and, after infecting them with a particular virus, simply track the phenotype of $\mathrm{T}$ cells expressing a $\mathrm{T}$ cell receptor (TCR) known to be specific for a particular virus epitope presented by a major histocompatibility (MHC) molecule known to be expressed in that mouse strain. Take that study into humans, and whole new dimensions of genetic and environmental complexity follow. Humans are outbred-and therefore express unknown combinations of human leukocyte antigen (HLA) molecules-and are generally kept outside pathogen-free facilities; as a result any group of individuals contains unknown varieties of $\mathrm{T}$ cells specific for unknown varieties of viral peptide-HLA complexes. Tackling human studies thus requires a quantum leap in our technological and computational sophistication.

Much of the complication stems from randomness-manifested most obviously at the genetic level-intrinsic to immune-system development. For example, whereas receptors that control activity of hepatocytes in the liver are encoded in the germ line and respond to the same ligands in all individuals, receptors that dictate responsiveness of lymphocytes are generated by somatic recombination among a pool of distinct germ-line gene segments; additional mutagenesis adds further randomness. As such, each individual harbors a different fleet of lymphocytes, each lymphocyte expressing a different receptor, each of which recognizes a different ligand. No two adults, even identical twins, have the same immune repertoire.

Fortunately, the past few years have brought marked progress in technologies that enable researchers to grapple with this heterogeneity and randomness, and generate the large datasets needed for high-resolution description of the immune system; computational approaches that enable extraction of useful insights from these datasets are also being developed. The state of the art of these methodologies is covered in a series of articles (http://www.nature.com/focus/high_dimensional_immune_analysis/) in the February issues of Nature Biotechnology and Nature Immunology.

On the translational side, these technologies may help generate new therapeutic reagents, improve the efficacy of existing ones and explain cryptic clinical observations. For example, high-throughput sequencing of the antibody repertoire can be used to isolate from patients rare pathogen-neutralizing antibodies that control infections; these antibodies might then be used as therapeutic agents to treat others or to help design new immunogens. Vaccine development might also be facilitated by knowledge of which pathogen epitopes could actually be seen by TCRs present in a majority of individuals in any given population. This sort of analysis is now possible, thanks to mass cytometry coupled with combinatorial peptide$\mathrm{MHC}$ tetramer staining. Knowledge of TCR repertoires might help explain seemingly random observations such as why some but not other cancer patients respond to immune system checkpoint-blockade therapy.

In terms of basic immunology, such technologies can reveal heterogeneity obscured by earlier bulk analyses. And this heterogeneity may be of sufficient magnitude so as to highlight new avenues of research and to call into question the relevance of some long-held assumptions. For example, a recent 35-parameter mass cytometry analysis of human natural killer (NK) cells revealed up to 30,000 different NK cell phenotypes in one person (Sci. Transl. Med. 5, 208ra145; 2013). Whether all 30,000 are distinct in terms of function and physiological importance remains unclear. What is clear, however, is that previous assumptions about immune-cell lineages falling neatly into a few definable 'subsets'-based largely on analyses of inbred mice-may simply not apply to humans.

The extent of heterogeneity in the human immune system means that enabling interlaboratory data sharing will be essential. Consider a study aimed at identifying which autoantibodies exacerbate human lupus. If the antibody repertoires of two healthy people and two lupus patients were sequenced, as a result of the randomness mentioned above the culprit antibody would be obscured among a sea of other antibodies found in the sick but not the healthy people; just as many differences might be seen between the two sick people. As such, studies intended to identify rare receptors or antibodies will require samples and data from more people than could reasonably be expected to be generated in one laboratory. Shared repositories with the capability to host properly annotated standardized data from different individuals will enable these large-scale studies. It is encouraging that some efforts to develop such standards and repositories are under way (Nat. Biotechnol. doi:10.1038/nbt.2777; 19 January 2014).

Clearly, human immunology is on the verge of an exciting new era. As the field increasingly adopts these new multidimensional approaches to characterizing the human immune system, we envisage a future where the types of translational and basic questions we ask are limited less by technology and more by our imaginations.

Nature Biotechnology and Nature Immunology are grateful to sponsor Adaptive Biotechnologies, whose support enables this focus to be freely available for the next 6 months. 